\title{
Am I Nearly There? The Effect of Anticipated Running Distance on Perceived Exertion and Attentional Focus
}

\author{
Denise A. Baden ${ }^{1}$, Lawrence Warwick-Evans ${ }^{1}$, and Julie Lakomy ${ }^{2}$ \\ University of Southampton
}

\begin{abstract}
Two studies tested the hypothesis that teleoanticipatory mechanisms regulate the perception of exertion (RPE) in the context of expected exercise duration by the adjustment of attentional focus. Study 1 involved 22 runners who participated in a short (8-mile) run and a long (10-mile) run on separate days. Pace did not differ between conditions $(M=6.3 \mathrm{mph})$. Runners reported on their attentional focus (proportion of associative to dissociative thoughts) and RPE at regular intervals. Study 2 involved 40 participants who ran twice on a treadmill at the same speed and gradient: once when they expected to run for $10 \mathrm{~min}$ (short condition) and once when they expected to run for $20 \mathrm{~min}$ (long condition). In both studies, RPE was lower throughout the long condition. In Study 1 there were more dissociative thoughts in the long condition. Study 2 showed the same trend, although the results were nonsignificant. In both studies RPE was inversely correlated with dissociative thoughts, supporting the hypothesis that runners pace themselves cognitively by manipulating their attentional focus.
\end{abstract}

Key Words: exercise, expectation, RPE, cognitive strategies, teleoanticipation

It is an accepted observation that fatigue, whether defined in terms of subjective ratings of perceived exertion (RPE) or in terms of diminishing performance, increases as exercise progresses. When we think of exercise related variables such as fatigue, we tend to think in terms of how far we are from the beginning. One might talk of being tired after a 5-mile run, for example. However, if the pace did not differ, would a runner still be as tired 5 miles into a 10 mile run? Could fatigue be partly a function of how far one is from the end as well as how far from the beginning?

Ulmer (1996) put forth the concept of teleoanticipation to describe distance perception or consideration of the finishing point. With reference to regulation of muscular metabolic rate during exercise, Ulmer theorized that there must be some kind of central programmer which focuses on the finishing point of the task and works backward from that point when regulating optimal metabolic rate and motor output. This was based in part on his finding that, when athletes were asked to maintain a constant RPE during exercise, work output declined over time, reached a plateau, and then increased at the end.

${ }^{1}$ Dept. of Psychology, and ${ }^{2}$ Dept. of Sports \& Health Studies, University of Southampton, Highfield, Southampton SO17 1BJ, U.K. 
The ability of individuals to gauge exercise intensity or to complete a test with similar times on separate occasions (Dunbar, Robertson, Baun, et al., 1992) endorses the notion of subconscious teleoanticipation regulation. This theory also received empirical support from a recent study demonstrating changes in psychological and performance variables attributable to expectations about the finishing point. Participants cycled on an ergometer for $8 \mathrm{~min}$ (short condition) and for 16 min (long condition). Heart rate (HR) was significantly higher throughout the 8-min condition when compared to the first $8 \mathrm{~min}$ of the 16-min condition. Pedal cadence was the same for both conditions, except that participants significantly sped up in the final minute of exercise in both conditions (Baden, 2002). It was suggested that the lower HR throughout the long condition could be attributable either to differences in motor efficiency or to psychological factors such as attentional focus.

Previous studies have shown that subjective feelings of fatigue can be influenced by expectations about task duration. Walster and Aronson (1967) found that participants engaged in a series of fatiguing visual tasks gave significantly higher ratings of fatigue on the third trial of a 3-trial session than on the third trial of a 5-trial session. They suggested that the participants repressed their feelings of fatigue until the last trial. Similarly, Rejeski and Ribisl (1980) found that RPE was higher for runners while running for $20 \mathrm{~min}$ on a treadmill than for those same runners when they believed they were to run for $30 \mathrm{~min}$. Based on Ulmer's theory of teleoanticipation (1996), a cognitive discussion model was put forth (St Clair Gibson, Lambert, Lambert, Hampson, \& Noakes, 2001) in which the subjective sensation of fatigue is the primary input into the central programmer and functions to avoid premature exhaustion by regulating exercise intensity, using prior experience as a guide.

It has been suggested that changes in RPE due to expectancies about task duration may be mediated via the participants' focus of attention during exercise (Rejeski, 1985). A landmark study by Morgan and Pollock (1977) into the types of thoughts engaged in by marathon runners gave rise to the proposal that there are two main cognitive strategies: association, by which runners focus on bodily signals, and dissociation, which distracts from bodily sensations by attention to the environment, daydreams, etc. Morgan and Pollock found that associative strategies were predominant in elite marathon runners, while subelite runners adopted a more dissociative strategy.

The theoretical underpinning of the proposed relationship between attentional focus and RPE was derived from the concept of attentional capacity (Leventhal \& Everhart, 1979). For example, Rejeski (1985) applied Leventhal and Everhart's parallel processing model of pain to concepts such as fatigue and perceived exertion. This model shows a distinction between perception and focal awareness, so that although we may perceive a whole array of external and internal sensory stimuli subconsciously, we will be conscious only of the stimuli we selectively attend to. The bottleneck in this flow of information is the point of focal attention, as this has the most limited capacity. The prediction arising from the concept of limited channel capacity is that anything which competes with cues arising from physical exertion in terms of occupying attentional focus will reduce RPE. Rejeski suggested that dissociative thoughts occupy limited channel capacity and thus prevent exertional cues from reaching one's level of awareness.

The majority of studies have supported the predictions arising from this theory, showing a negative relationship between RPE and dissociative strategies (Nethery, Harmer, \& Taaffe, 1991; Pennebaker \& Lightner, 1980; Schomer 1986) and a 
positive relationship between endurance and dissociative strategies (Asmussen \& Mazin, 1978; Gill \& Strom, 1985; Morgan, Horstman, Cymerman, \& Stokes, 1983; Weinberg, Smith, Jackson, \& Gould, 1984). No studies have been undertaken, as far as we know, that examine how both RPE and attentional focus change over time in relation to expected exercise duration.

St Clair Gibson, Lambert, and Noakes (2001) suggested that when there is no set exercise duration and participants continue until they feel they fatigued, cessation of exercise may result in part from mental fatigue; they proposed that higher mental functions such as concentration and motivation may carry a metabolic cost and lead to the perception of fatigue. Studies on self-control support this as a plausible hypothesis. For example, a series of studies showed that when participants were asked to exert self-control and resist temptation, their subsequent motivation to persist at an unsolvable puzzle was reduced (Baumeister, Bratslavsky, Muraven, \& Tice, 1998). In a later summary of such studies, Baumeister, Muraven, and Tice (2000) concluded that acts of volition and self-control draw on a shared resource that is limited but renewable, and claimed that there are mechanisms for conserving these higher mental functions. Accordingly, just as a central programmer is proposed to balance physical energy reserves with expected physical costs by adjusting motor output, it may be that psychological processes also require conservation.

We set out to test the hypothesis that individuals pace themselves mentally. Two studies compare how RPE and attentional focus change over time during a short run and a long run, where differences in pace are kept to a minimum (Study 1) or pace is kept constant (Study 2). The first study favors ecological validity and examines experienced runners in a natural outdoor setting. The second study uses two kinds of participants and favors tighter control over extraneous variables, employing a treadmill so that speed and gradient can be fixed. It is predicted that RPE will be suppressed when a long run is expected, as participants use a more dissociative strategy to distract themselves from fatigue (Rejeski, 1985). If this is so, then both RPE and the percentage of associative thoughts should be lower throughout the long run than the short run, and should be correlated with each other within each run. With regard to attentional focus over the course of the run, the established correlation between RPE and associative thoughts (Schomer, 1986; Tenenbaum, 2001) would lead one to expect that the proportion of associative thoughts would rise over time alongside RPE. Similarities in the results between the two studies and among the participant types would argue for the effects of mental pacing being ubiquitous and relatively independent of setting and participant type.

\section{STUDY 1 \\ Method}

\section{Participants and Measures}

Twenty two members of a running club, 14 men and 8 women with a mean age of 48 years $(S D=8.9)$, volunteered to take part in the study. Their typical run length ranged between 5 and 12 miles $(M=8.86, S D=2.2)$, and all had completed the runs that were to be done as part of the study at least once before. Each participant gave informed consent and the the study was approved by the Joint Ethical Committee of the University of Southampton.

Speed. Although speed was not one of the variables under consideration, it was important to determine to what extent the pace differed between the two 
conditions, as this would in turn have an impact on the other dependent variables. Mean speed over each section was calculated by the time taken to complete each section of the run.

RPE. The RPE scale (Borg, 1973) begins at 6, "no exertion at all," and goes up to 20, "maximal exertion." The odd numbered categories have verbal anchors (e.g., $11=$ fairly light, $13=$ somewhat hard, $15=$ hard, $17=$ very hard). Reliability and validity was first established in 1973 (Skinner, Hutsler, Bergsteinova, \& Buskirk), and the scale has since been widely used in research and in clinical and training settings to provide a subjective supplement to physiological data recorded during exercise. Participants were briefed in the use of the RPE scale, and all indicated that they felt confident with its use prior to the run.

Attentional Focus. Many studies have used retrospective methods to assess attentional focus, such as interviews (Morgan \& Pollock, 1977), thought checklists (Okwumabua, Meyers, Schleser, \& Cooke, 1983), bipolar scale (Tammen, 1996), running strategies questionnaire (Silva \& Appelbaum, 1989), forced-choice questionnaire (Acevedo, Dzewaltowski, \& Gill, 1992), and race diaries (Masters \& Lambert, 1989). However, to the extent that these studies rely on retrospective data, they may not give an accurate picture of the types of thoughts present throughout the run. Yet, attempting to avoid retrospective falsification by recording thoughts of runners as they occur by having runners talk into a dictaphone while running (Schomer, 1986) may bias them toward associative thoughts, since dissociative thoughts are inherently more unconscious and may disappear if explicitly verbalized. For example, a study that had a researcher cycle alongside runners to ask them about their thoughts found that the runners tended to report associative thoughts freely, but had trouble remembering or verbalizing dissociative thoughts, although they acknowledged engaging in them (Sacks, Milvy, Perry, \& Sherman, 1981).

As it was of particular interest to look at the relative proportion of associative to dissociative thoughts over the course of the run, we decided to favor temporal proximity and noninvasiveness of technique over detail. We therefore adopted the method used by Tammen (1996) of having participants rate on a 10-cm bipolar line to what extent their thoughts were primarily associative or dissociative throughout the section of the run just completed. The simplicity and brevity of this method also allowed the runners to maintain their stride as far as possible, with minimum interference in their usual run. Participants were fully briefed in the distinction between associative and dissociative thoughts, and all completed a brief quiz before beginning the run to ensure that they were comfortable with the distinction (Appendix). The instructions given were as follows:

At regular intervals I will ask you to put a cross on the line below that represents the approximate ratio of associative thoughts to dissociative thoughts over the last segment of the run. But please don't think that you have to be aware of exactly what you are thinking all the time, an approximate proportion is fine. Also, quite often dissociative-type thoughts are like daydreams and quickly forgotten. So although you may start off by thinking that your feet hurt, you may then go on to speculate whether to get new trainers and then may find you are in a daydream you may not remember. In this case, just the original thought counts as an associative thought and the rest is dissociative.

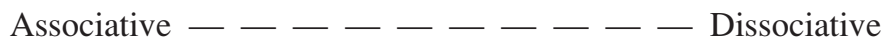


The data are presented in terms of percentage of associative thoughts, so that a cross halfway on the bipolar scale is interpreted as $50 \%$ associative and $50 \%$ dissociative, and a cross three-quarters of the way toward the associative pole is interpreted as $75 \%$ of thoughts being associative and $25 \%$ being dissociative. Visual assessment of the rating was assisted by the spaces separating the 10 dash clusters that make up the line as shown.

\section{Design and Procedure}

The proposed study was developed with the cooperation of the organizer of the running club, who agreed to allow us to approach the members with regard to taking part in the study. All club members received an information pack giving details of the study, together with forms for them to complete providing personal information such as age, gender, previous and current running and exercise activities, and a consent form. The pack also provided full details of the Borg RPE scale and the associative/ dissociative scale. The experiment took place during the club's regular evening run in the New Forest, and all members who were available to run on both occasions agreed to participate.

We had originally planned to compare a 5-mile and a 10-mile run. However, preliminary talks with the organizer of the running group indicated that such different distances would yield different schemata and pacing strategies on a large scale, whereas if the two runs differed by only $20 \%$, the members would all run together as a group as usual, with simply an additional diversion for the 10-mile run. It was therefore decided to use 8 and 10 miles as the short and long condition, as these distances were at the upper limit of the running club's normal runs.

The study used a within-subjects design: each participant ran 8 miles one week (short condition) and 10 miles another week (long condition), the order being counterbalanced. On the second run we asked the participants if they felt as fit as they had the week before, and all said they felt the same. The time and weather conditions were similar for both runs: sunny and warm, approx. $20^{\circ} \mathrm{C}$. The two runs were the same except that the long run deviated at 7.25 miles for a detour of 2 miles before joining back up, so that the final stretch was the same for both runs. Training effects were not a concern, as all participants were regular runners and had often taken part in similar runs. However, the novelty effect of taking part in an experiment was an issue. This was minimized by adhering as closely as possible to their usual procedure. The meeting time and place was the same as on usual running nights, and all participants were familiar with the course.

In the running club were several subgroups of runners who were used to running with the same small cluster of people of similar ability each week. Random allocation to the long and short condition was carried out within each cluster of runners, so that the pace for both conditions would be more likely to remain the same on both occasions. Prior to the run, the participants were briefed once again as a group and then individually on the use of the Borg RPE scale and associative/dissociative scale. The participants began running. At 2 miles, 3.75 miles, 5.5 miles, 7.25 miles, and (on the long run) 9.25 miles, they stopped briefly at a table to record their RPE and proportion of associative to dissociative thoughts. Runners returned the following week at the same time to repeat the run, this time doing the long run if they had already done the short run, or vice versa. They were asked to refrain from excessive alcohol or exercise for $48 \mathrm{hrs}$ prior to each run. 


\section{Statistical Treatment}

Two-way repeated-measures ANOVAs were conducted. The dependent variables were RPE and percentage of associative thoughts. The within-subjects factors were condition with two levels (short $=8$ miles, long $=10$ miles) and distance with four levels ( 2 miles, 3.75 miles, 5.5 miles, and 7.25 miles). The Greenhouse-Geisser correction was used when the assumption of sphericity was violated. An alpha level of $p<.05$ was established. Effect size is presented in terms of eta squared $\left(\eta^{2}\right)$. Pearson's product-moment correlations were performed so as to determine the relationships between RPE and the percentage of associative thoughts at each time point.

\section{Results}

We conducted preliminary analyses using gender and order as between-subjects factors, checking for significant interactions for each factor individually for each dependent variable. No significant interactions with condition or distance were found for either factor for any of the dependent variables, and so these factors have been excluded from the analyses.

Speed. The pace throughout the short condition did not differ significantly from that of the long condition: $M=6.32$ vs. $6.26 \mathrm{mph}, S D=0.54 \mathrm{vs}$. 0.42 , respectively. No significant correlations were found between speed and any of the other variables at any time point. Mean correlations of all time points were as follows: speed and RPE, mean $r(21)=-.11$; speed and associative thoughts, mean $r(21)=.08$.

$R P E$. The condition main effect was significant, $F(1,20)=4.36, p<.05$, $\eta^{2}=.18$, with RPE being higher in the short condition than in the long condition: $M=13.59$ vs. $13.36, S D=1.63$ vs. 1.42 , respectively, as shown in Figure 1 . The distance main effect was also significant, with RPE increasing over distance, $F(3$, $18)=18.34, p<.001, \eta^{2}=.37$. There was no significant Condition $\times$ Distance interaction.

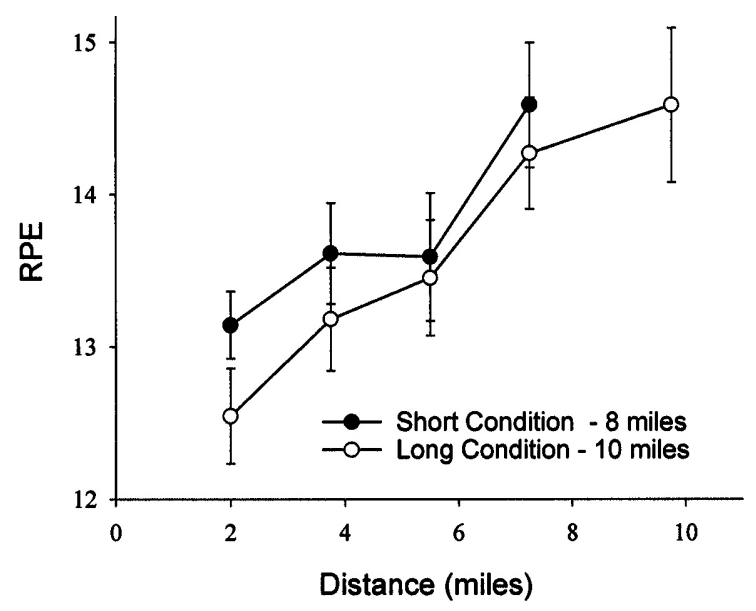

Figure 1 - Mean $(N=22)$ RPE $( \pm S E)$ throughout a short and a long cross-country run as a function of expected distance in Study 1. 


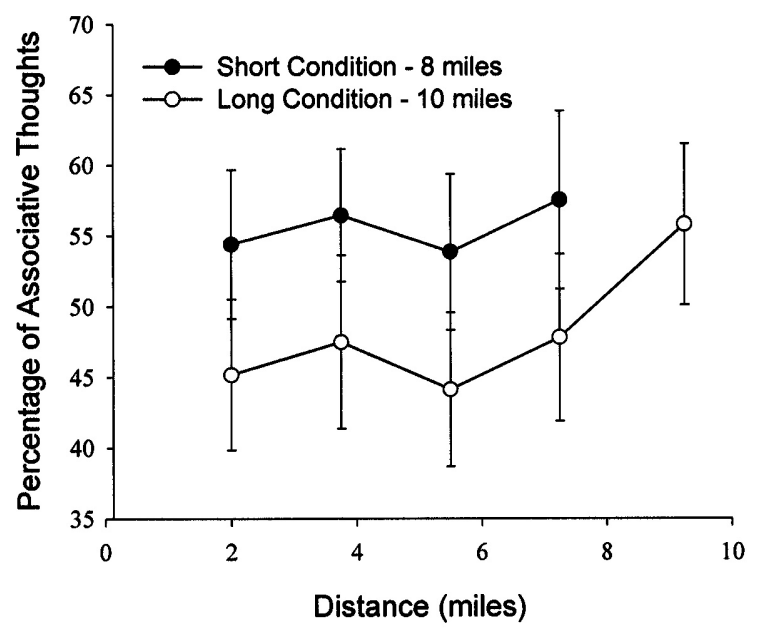

Figure 2-Mean $(N=22)$ percentage of associative thoughts $( \pm S E)$ throughout a short and a long cross- country run as a function of expected distance in Study 1.

Attentional Focus. The condition main effect was significant, $F(1,21)=$ $6.85, p<.02, \eta^{2}=.25$, with the percentage of associative thoughts being higher in the short condition than in the long condition: $M=55.57 \mathrm{vs} .46 .16, S D=21.15 \mathrm{vs}$. 24.70, as shown in Figure 2. The main effect of distance was nonsignificant, and so was the Distance $\times$ Condition interaction.

\section{Relationship Between Variables}

Pearson's product-moment correlations indicated a positive relationship between RPE and associative thoughts that grew stronger over distance and was significant at the end of both runs (Table 1).

\section{Discussion}

RPE was higher all the way through the short run compared to the long run, supporting the hypothesis that expectancies concerning task duration affect RPE. The fact that speed did not differ significantly between the two conditions, together with the minimal (and inverse) correlation between RPE and speed ( $r=-.11)$, suggests that the higher RPE in the short condition was unlikely to be due to a faster pace.

It was suggested that there is a link between dissociative thoughts and RPE whereby runners use a dissociative strategy to distract themselves from fatigue (Rejeski, 1985). According to an information-processing perspective, this occurs as dissociative thoughts occupy the attentional space and leave less room for physical sensations to be processed. The data support this explanation, as Figure 2 indicates a lower level of associative thoughts throughout the long run. Again, the lack of any correlation with speed suggests that this result cannot be attributed to differences in pace. In further support of a link between attentional focus and RPE are the positive correlations between RPE and associative thoughts (Table 1), which 
Table 1 Correlation Table, RPE, and Percentage of Associative Thoughts in Study 1

\begin{tabular}{lcc}
\hline Distance & Short condition & Long condition \\
\hline 2 & -.06 & -.05 \\
3.75 & .22 & .33 \\
5.5 & .24 & .32 \\
7.25 & $.52^{*}$ & $.43^{*}$ \\
9.25 & $\mathrm{n} / \mathrm{a}$ & $.44^{*}$ \\
\hline
\end{tabular}

* Significant correlations, $p<.05$.

reach significance on the final measurements. Whether the direction of attentional focus is the cause or the effect of RPE, however, remains unclear. The correlation may indicate that when people are working hard in terms of perceived effort, their thoughts naturally become more associative (Tenenbaum, 2001). Alternatively, it may be that the correlation between RPE and associative thoughts reflects strategies for manipulating attentional focus.

Although there was a higher level of associative thoughts throughout the short run, the pattern of attentional focus in both runs was the same. In both cases associative thoughts increased initially, then dipped in the middle and then were highest at the end of the run. The symmetry between the two conditions is striking and a tentative hypothesis is suggested: RPE is a psychophysiological construct; just as the physical components of the sense of effort are controlled by adjusting exercise intensity, it may be that the psychological components of RPE are regulated in part by attentional focus. For example, the pace and intensity of exercise will be set by each runner depending on situational and dispositional factors. One would expect a runner to adopt a very different baseline pace for a 1,000-m run than for a 10,000-m run. However, within each run the pattern of speed may be consistent, falling at the beginning, plateauing out, and then increasing at the end. It may be that the psychological components of RPE can be similarly manipulated by cognitive strategies.

With regard to attentional focus, this may be apparent in the present study by the overall lower level of associative thoughts during the long run, together with the consistent pattern of associative thoughts over the course of both the short and long runs. Follow-up studies that manipulate expected running distance and measure RPE and attentional focus are needed to test this hypothesis.

A potential confound in this study was the fact that the participants ran in their own small groups, often on the basis of preferred pace (although this was not measured). It is possible, therefore, that particular individuals in each group may in effect have set the pace. Social discourse within groups may also have affected attentional focus and RPE. A further limitation of the study was the fact that the difference between the short and long conditions was just $20 \%$. Accordingly, in the next study the expected length of the long condition was twice that of the short condition, and participants ran individually in a controlled environment where speed, distance and gradient could be precisely controlled. 


\section{STUDY 2}

In this study the participants ran on a treadmill at a predefined speed and gradient for 10 min under two conditions a week apart: once when they expected to run for $10 \mathrm{~min}$, and once when they expected to run for $20 \mathrm{~min}$ but were stopped unexpectedly at $10 \mathrm{~min}$. This study compared young, healthy participants with individuals who had been prescribed exercise by their physician and who exercised regularly as part of a local Active Options program. If similar effects were found for each participant group, one could be more confident that anticipatory effects were not simply confined to athletic individuals.

\section{Method}

\section{Participants}

Participants comprised two groups: (a) healthy individuals, ages 18-30, who were recruited from a sports degree program at Southampton University to participate in an experimental study; and (b) individuals in an Active Options program who had been prescribed exercise by their physician, typically because they were deemed to be at risk of CHD (e.g., due to high blood pressure, high cholesterol levels, angina or myocardial infarction). Other reasons for being in Active Options included stroke, obesity, and arthritis. The exclusion criteria for participation were: being on beta blocker medication, having blood pressure higher than 160/100, body mass index greater than 32 , and uncontrolled angina or diabetes. Individuals in Active Options are prescribed a set routine of exercise which is updated every 8 sessions. After 16 sessions they are termed graduate patients. This study involved only graduate patients. Each participant gave informed consent and the Joint Ethical Committee of the University of Southampton approved the study. Participant characteristics are presented in Table 2 .

\section{Materials and Measures}

Study 2 made use of the Life Fitness 6500 HR self-inclining programmable automated treadmill. It allows users to program the speed, gradient, and duration of the session, and also adds a 1-min cooldown period afterward at zero gradient and reduced speed. The measures included Borg's RPE scale (Borg, 1973; see Study 1 for details).

Table 2 Participant Characteristics, Study 2: Active Options vs. Young

\begin{tabular}{|c|c|c|c|c|c|c|c|}
\hline \multirow{2}{*}{$\begin{array}{l}\text { Participant } \\
\text { type }\end{array}$} & \multirow[b]{2}{*}{$N$} & \multicolumn{2}{|c|}{$\underline{\text { Age }}$} & \multirow{2}{*}{$\begin{array}{c}\text { Men } \\
n\end{array}$} & \multirow{2}{*}{$\begin{array}{c}\text { Women } \\
n\end{array}$} & \multicolumn{2}{|c|}{ Exercise score } \\
\hline & & $M$ & $S D$ & & & $M$ & $S D$ \\
\hline Active Options & 22 & 65.0 & 5.95 & 10 & 12 & 3.75 & 1.92 \\
\hline Young & 18 & 21.28 & 1.74 & 10 & 8 & 4.17 & 3.12 \\
\hline All Participants & 40 & 45.32 & 22.49 & 20 & 20 & 3.94 & 2.51 \\
\hline
\end{tabular}

Note : Exercise Score = number of hours per week specifically engaged in exercise. 
Attentional Focus. Participants were verbally briefed on the distinction between associative and dissociative thoughts and were given an explanatory sheet incorporating a brief quiz (see Appendix) to ensure that they understood the terms correctly. Participants reported at regular intervals to what extent they were focusing on the body (associative) or had distracting (dissociative) thoughts during the section of the run just completed. They estimated what percentage of their thoughts were associative; all other thoughts were deemed dissociative. Five of the Active Options participants did not feel confident about making the distinction, and therefore data on their percentage of associative thoughts were excluded.

\section{Procedure}

The experiments took place in a public gym, although the Active Option sessions in the gym are closed to other members of the public during this time. The participants were familiar with the exercise and the setting. A within-subjects design was used, the speed and gradient was the same for both conditions, and all conditions were counterbalanced.

Introductory Session. Participants were briefed on the procedure. The Borg RPE scale and the associative/dissociative thought scale were discussed with the participants to ensure that they fully understood these measures, and general information was taken. The speed and gradient to be used for the experimental sessions were defined, and it was made clear that the same speed and gradient would apply to both sessions. Dates were set for the experimental sessions, and participants were asked to refrain from excessive alcohol or exercise for 48 hours prior to each session.

Experimental Sessions. In each session the Active Options participants did their normal prescribed exercise routine but reduced it by $10 \mathrm{~min}$, typically by cutting out the usual treadmill segment of their routine. This was to ensure that the experimental session on the treadmill would not take them beyond their prescribed level of exercise. Those in other group were allowed to exercise prior to the experiment as they wished, based on their usual exercise activity. The main criteria for both groups were that they did the same pretreadmill routine for both conditions and that the experimental session on the treadmill would be the final exercise they would do in that gym session. Before starting, the participants rested for $10 \mathrm{~min}$ while the procedure and measures were briefly reviewed. On the second run a week later, the participants were asked if they felt as fit as they had the week before; all indicated that they felt the same.

Short Condition. The participants programmed the treadmill to run for 10 min at their usual speed and gradient. During the run they gave verbal estimates of their RPE and percentage of associative thoughts over the previous segment at 3, 5, 7, and 9 min into exercise. Just before these measurements they were informed as to how far they had yet to go. For example, at 9 min they were told they were nearly finished. At 10 min the treadmill automatically slowed down, and a cooldown minute followed at zero gradient and reduced speed.

Long Condition. This was the same as the short condition except that the participants expected to run for $20 \mathrm{~min}$. They stood on the treadmill and were distracted while the experimenter programmed the treadmill to run for $10 \mathrm{~min}$ at their usual speed and gradient. RPE and the percentage of associative thoughts were recorded at $3,5,7$, and 9 min into exercise. Prior to each measurement the participants were 
informed as to how far they had yet to go; for example at 9 min they were told they were nearly halfway through. At $10 \mathrm{~min}$ the treadmill slowed down and continued for a further minute at zero gradient and reduced speed before stopping. When the participants queried the unexpected slowing of the treadmill, they were told there had been an error in setting the duration of the exercise but that enough data had already been collected anyhow. Afterward participants circled "yes," "no," or "not sure" to the question "Did you believe you were going to be on the treadmill for 20 minutes?" All responded yes, indicating that the manipulation had worked and they had been genuinely deceived with respect to the duration of the run.

\section{Statistical Analyses}

Two-way repeated-measures ANOVAs were conducted as with Study 1, except that the within-subjects factors were the experimental condition with two levels $($ short condition $=$ expect $10 \mathrm{~min}$, long condition $=$ expect $20 \mathrm{~min}$ ) and time with four levels $(3,5,7$, and 9 min). The dependent variables were RPE and percentage of associative thoughts.

\section{Results}

We conducted preliminary analyses using gender, order, and participant type as between-subjects factors, checking for significant interactions for each factor individually for each dependent variable. No significant interactions with condition or time were found for any of these factors for any of the dependent variables, and so these factors have been excluded from the analyses.

$R P E$. The condition main effect was significant, $F(1,38)=8.03, p<.01, \eta^{2}$ $=.17$, with RPE being higher in the short condition than in the long condition: $M$

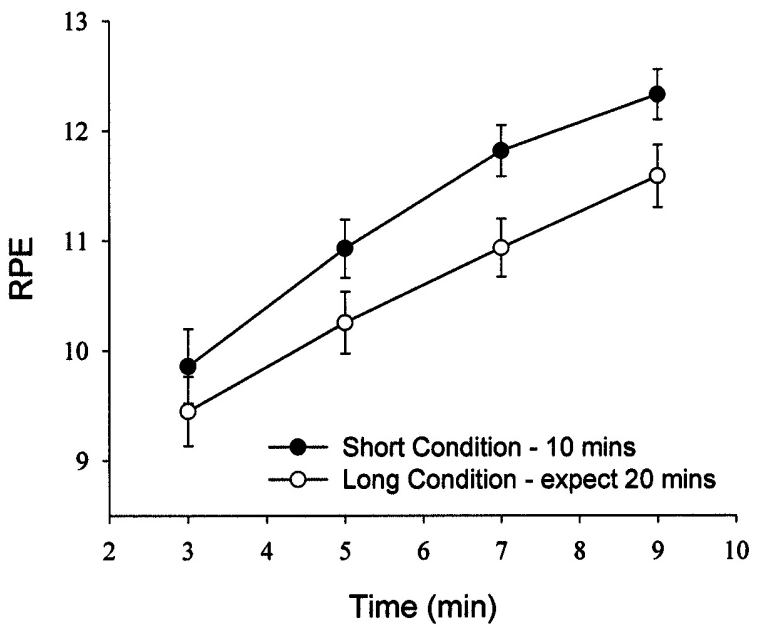

Figure 3 - Mean $(N=40)$ RPE $( \pm S E)$ throughout two treadmill sessions of identical workloads as a function of expected run duration in Study 2. 


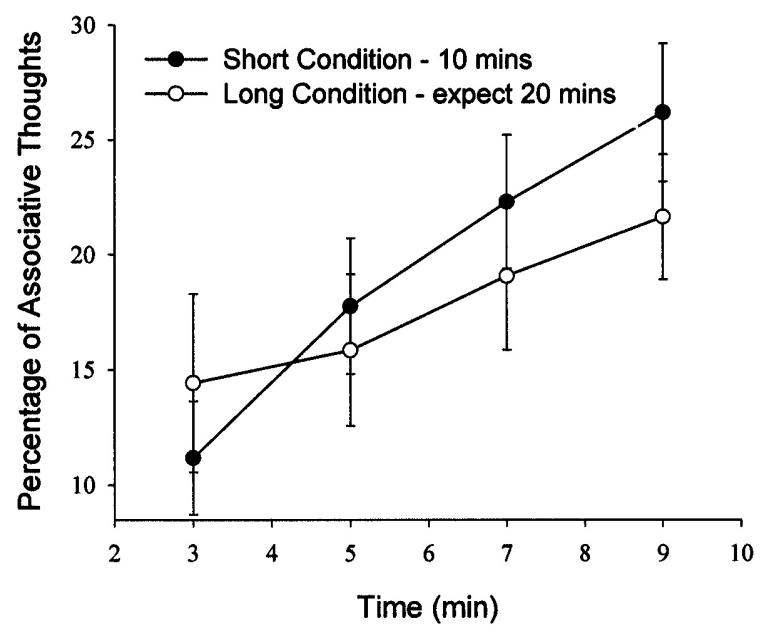

Figure $4-$ Mean $(N=40)$ percentage of associative thoughts $( \pm S E)$ throughout two treadmill sessions of identical workloads as a function of expected run duration in Study 2.

$=11.24$ vs. $10.56, S D=0.24$ vs. 0.27 , respectively, as shown in Figure 3 . The time main effect was significant, $F(3,36)=33.06, p<.001, \eta^{2}=.68$, with RPE increasing over time. The Condition $\times$ Time interaction effect was nonsignificant.

Percentage of Associative Thoughts. The condition main effect was nonsignificant. However, as shown in Figure 4, the trend was in the expected direction with the percentage of associative thoughts being higher in the short condition than in the long condition: $M=19.37$ vs. $17.76, S D=2.37$ vs. 2.96 , respectively. The time main effect was significant, $F(3,32)=6.31, p<.01, \eta^{2}=.25$, with the percentage of associative thoughts increasing over time. The Condition $\times$ Time interaction effect was not significant once the Greenhouse-Geisser correction was used to adjust for violation of the sphericity assumption $(p=.07)$.

\section{Relationship Between Variables}

A positive correlation was found between RPE and percentage of associative thoughts in both conditions and reached significance at the end (Table 3 ). To further explore this relationship, we computed new variables that were the difference in RPE between each condition at each time point, and the difference in percentage of associative thoughts between each condition at each time point. For instance, RPE diff. at 3 min was created by subtracting RPE at $3 \mathrm{~min}$ in the long condition from $\mathrm{RPE}$ at $3 \mathrm{~min}$ in the short condition, so RPE diff. is a measure of the difference between the two conditions. Correlation analyses between the difference in RPE between both conditions at each time point and the difference in the percentage of associative thoughts between both conditions indicated a significant positive correlation at 7 and $9 \mathrm{~min}$ (Table 3 ). 
Table 3 Correlation Coefficients for RPE \& Percentage of Associative Thoughts and for Differences in RPE and in Percentage of Associative Thoughts in Study 2

\begin{tabular}{|c|c|c|c|}
\hline Time & $\frac{\text { RPE \& \% }}{\text { Short }}$ & $\frac{\text { c. thoughts }}{\text { Long }}$ & $\frac{\text { RPE diff. \& \% Assoc. diff. }}{\text { Short - Long }}$ \\
\hline $3 \mathrm{~min}$ & .25 & .22 & -.02 \\
\hline $5 \mathrm{~min}$ & .29 & .17 & .06 \\
\hline $7 \mathrm{~min}$ & $.38 *$ & .21 & $.36^{*}$ \\
\hline $9 \min$ & $.42 *$ & $.45^{*}$ & $.38 *$ \\
\hline
\end{tabular}

Note RPE diff. $=$ RPE in short condition minus RPE in long condition. \% Assoc. diff. $=$ $\%$ of associative thoughts in short condition minus $\%$ of same in long condition.

* Significant correlations, $p<.05$.

\section{Discussion}

With respect to RPE, this study replicated the results of Study 1 and found a significantly higher RPE throughout the short condition. It was hypothesized in Study 1 that the lower RPE throughout the long condition may be due to an increased use of dissociative strategies that distract attention from the physical symptoms accompanying exercise. This study did not find significantly more dissociative thoughts in the long condition, although the trend was in the predicted direction. However, RPE and the percentage of associative thoughts were positively correlated throughout the exercise, and the correlations were significant at the end of both conditions. In addition, significant positive correlations were found between the difference in RPE and in the percentage of associative thoughts on the last two measurements at 7 and 9 min into exercise (Table 3), consistent with the hypothesis that RPE can be suppressed by dissociative strategies.

The previous study found that the pattern of attentional focus was the same for both runs, although there was a higher level of associative thoughts throughout the short run. It was suggested that cognitive strategies may be used to control attentional focus similar to the way pacing strategies are used to control fatigue. This study did not show such a marked similarity between conditions, although both conditions showed a linear increase in the percentage of associative thoughts over time, with the percentage increasing more steeply in the short condition. However, this study involved just 10 min of exercise, compared to about 90 min in Study 1, and so there may not have been enough time for such patterns to emerge.

A possible confounding variable with respect to the Active Options participants was conversation. Although they had been told there should be no talking throughout the exercise except to report measurements, some could not help talking. With such participants, however, the levels of conversation were similar for each condition. Another potential confounding factor in this study was the fact that the two groups differed both in age and health status. Although it is to be expected that the two groups would differ in their absolute responses to the exercise in terms of exercise intensity, heart rate, etc., there was no a priori reason to suppose this 
would interact with the main effect of expected run duration at submaximal workloads. Indeed, an interesting finding was that participant group had no bearing on the results. Although the Active Options group was very different from the Young group in age and general health, the two groups did not differ with respect to their responses to differences in expected task duration.

\section{GENERAL DISCUSSION}

The results support the contention that perceptions of exertion are subject to teleoanticipatory mechanisms. Significant main effects of expected duration on RPE were found in both studies. For instance, participants who had run for $9 \mathrm{~min}$ and thought their run was nearly over felt significantly more exerted than when they had run for $9 \mathrm{~min}$ and thought they were less than halfway through. Bearing in mind the close dependence of RPE on work output (Skinner et al., 1973), one would not expect large effect sizes for purely psychological inputs. Hence, although the differences in RPE between the short and long conditions were not large $\left(\eta^{2}=.18\right.$ in Study $1 ; \eta^{2}=.17$ in Study 2), we argue that they are nevertheless meaningful.

The proposal that the lower RPE in the long condition is due to attentional capacity being occupied by dissociative thoughts, diverting attention away from purely physical cues (Rejeski, 1985), is also supported. Study 1 found a significantly higher percentage of associative thoughts throughout the short run, together with a positive correlation between RPE and associative thoughts. In Study 2, although the differences in percentage of associative thoughts between both conditions was not significant, the trend was in the same direction, and there was a significant positive correlation between RPE and the percentage of associative thoughts, and also between the differences in RPE and associative thoughts. In both studies these correlations tended to get stronger over time and became significant only in the final few measurements.

We measured attentional focus throughout exercise by the use of a bipolar scale in Study 1, and by asking participants to report on their percentage of associative thoughts in Study 2. The brevity of this method allowed attentional focus to be measured during exercise without lengthy stops in the middle of exercise to fill out questionnaires. One drawback of this method, however, is that it assumes thoughts can be divided into associative, i.e., relating to the body and the exercise, or dissociative, i.e., everything else. Although most participants seemed to have no trouble accepting this concept and reporting on the relative proportion of associative thoughts, some found it difficult. In particular some Active Options participants in Study 2 did not feel they could capture their thought processes with a percentage. One claimed to have both associative and dissociative thoughts at the same time but at different levels of consciousness. Although measurements of attentional focus were not recorded for those who had trouble with this measurement, such comments suggest weaknesses in the scale.

The pattern of results for both studies is consistent with the notion of RPE being a regulated variable reminiscent of more physical variables. Many psychological constructs that depend in part on motivational factors have been shown to have a limited capacity. For example, self-control has been demonstrated to operate as though it were a limited resource (Baumeister et al., 1998; 2000). If RPE is seen in this light, it may be that within each situational/dispositional construct is an optimal level of ability/desire to expend effort. Individuals may naturally attempt 
to reach this optimum level of effort expenditure, so that a 10-mile run would yield RPE ratings proportionally lower than an 8-mile run, and this may be mediated by attentional focus. In addition, the fact that gender and participant type did not show any interactions with condition argues for these teleoanticipatory effects being ubiquitous and not confined to any one type of individual.

A case can thus be made for extending Ulmer's theory of teleoanticipation (1996) to incorporate the notion of psychological pacing throughout exercise. However, it is possible that Ulmer's proposal of a central programmer that regulates metabolic output in the context of expected duration is sufficient to account for the findings. The lower RPE throughout the longer runs, for instance, might reflect greater running economy rather than differences in attentional focus. For example, a previous study found that HR was lower when participants were expecting a longer exercise duration, and it was suggested that this might be due to greater motor efficiency in the long condition (Baden, 2002).

In conclusion, it is suggested that psychological variables such as RPE and attentional focus may not have limitless capacity, and the teleoanticipatory mechanisms put forth by Ulmer (1996) should be extended to include regulation of mental as well as physical output. Considering exercise variables purely from the perspective of amount of work completed is to ignore the attentional focus of the exerciser, whose thoughts may be fixed not on what he or she has done but on what he/she has left to do. Given that most sports have a defined endpoint or an expected duration, it may be worth considering teleoanticipatory expectations when measuring or attempting to manipulate psychological, physiological, and performance variables associated with sports and exercise.

Individuals such as coaches or aerobics trainers who try to encourage others during exercise tend to draw the exercisers' attention to the endpoint with phrases such as "nearly there," "just 5 more minutes," "one more mile and then you're done." It is not uncommon for these statements to be exaggerated, and sometimes completely false, in the encourager's attempts to increase motivation and performance. However, bearing in mind the lower RPE found when a longer duration was expected, any resulting short-term increases in pace may be counterproductive if the end is not immediately forthcoming, and thus it might be better to assure the exercisers that they still have a long way to go.

\section{References}

Acevedo, E.O., Dzewaltowski, D.A., \& Gill, D.L. (1992). Cognitive orientations of ultramarathoners. The Sport Psychologist, 6, 242-252.

Asmussen, E., \& Mazin, B. (1978). A central nervous component in local muscular fatigue. European Journal of Applied Physiology, 38, 9-15.

Baden, D.A. (2002). Goals and expectancies: Psychological and physiological effects of anticipating the end. Unpublished master's thesis, University of Southampton, UK.

Baumeister, R.F., Bratslavsky, E., Muraven, M., \& Tice, D.M. (1998). Ego depletion: Is the active self a limited resource? Journal of Personality and Social Psychology, 74, 1252-1265.

Baumeister, R.F., Muraven, M., \& Tice, D.M. (2000). Ego depletion: A resource model of volition, self-regulation, and controlled processing. Social Cognition, 18, 130-150.

Borg, G. (1973). Perceived exertion: A note on history and methods. Medicine and Science in Sports and Exercise, 5, 90-93. 
Dunbar, C.C., Robertson, R.J., Baun, R., Blandin, M.F., Metz, K., Burdett, R., \& Goss, F.L. (1992). The validity of regulating exercise intensity by ratings of perceived exertion. Medicine and Science in Sports and Exercise, 24, 94-99.

Gill, D.L., \& Strom, E.H. (1985). The effect of attentional focus on performance of an endurance task. International Journal of Sport Psychology, 16, 217-223.

Leventhal, H., \& Everhart, D. (1979). Emotion, pain and physical illness. In C.E. Izard (Ed.), Emotions in personality and psychopathology (pp. 263-298). New York: Plenum.

Masters, K.S., \& Lambert, M.J. (1989). The relations between cognitive coping strategies, reasons for running, injury, and performance of marathon runners. Journal of Sport \& Exercise Psychology, 11, 161-170.

Morgan, W.P., Horstman, D.H., Cymerman, A., \& Stokes, J. (1983). Facilitation of physical performance by means of a cognitive strategy. Cognitive Therapy and Research, 7, 251-264.

Morgan, W.P., \& Pollock, M.L. (1977). Psychologic characterization of the elite distance runner. Annals of the NY Academy of Sciences, 301, 382-403.

Nethery, V.M., Harmer, P.A., \& Taaffe, D.R. (1991). Sensory mediation of perceived exertion during submaximal exercise. Journal of Human Movement Studies, 20, 201-211.

Okwumabua, T.M., Meyers, A.W., Schleser, R., \& Cooke, C.J. (1983). Cognitive strategies and running performance - An exploratory study. Cognitive Therapy and Research, 7, 363-370.

Pennebaker, J.W., \& Lightner, J.M. (1980). Competition of internal information in an exercise setting. Journal of Personality and Social Psychology, 39, 165-174.

Rejeski, W.J. (1985). Perceived exertion - An active or passive process? Journal of Sport Psychology, 7, 371-378.

Rejeski, W.J., \& Ribisl, P.M. (1980). Expected task duration and perceived effort: An attributional analysis. Journal of Sport Psychology, 2, 227-236.

Sacks, M.H., Milvy, P., Perry, S.W., \& Sherman, L.R. (1981). Mental status and psychological coping during a 100-mile race. In M.H. Sacks \& M.L. Sachs (Eds.), Psychology of running (pp. 166-175). Champaign, IL: Human Kinetics.

Schomer, H. (1986). Mental strategies and the perception of effort of marathon runners. International Journal of Sport Psychology, 17, 41-59.

Silva, J.M., \& Appelbaum, M.I. (1989). Association dissociation patterns of United States Olympic marathon trial contestants. Cognitive Therapy and Research, 13, 185-192.

Skinner, J.S., Hutsler, R., Bergsteinova, V., \& Buskirk, E.R. (1973). The validity and reliability of a rating scale of perceived exertion. Medicine and Science in Sports and Exercise, 5, 94-96.

St Clair Gibson, A., Lambert E.V., Lambert, M.I., Hampson, D.B., \& Noakes, T.D. (2001). Exercise and fatigue control mechanisms. International SportMed Journal, 2(3), xxxx. IIAu: need page nos. Also, called International Sports Medicine Journal?

St Clair Gibson, A., Lambert, M.I., \& Noakes, T.D. (2001). Neural control of force output during maximal and submaximal exercise. Sports Medicine, 31, 637-650.

Tammen, V.V. (1996). Elite middle and long distance runners associative dissociative coping. Journal of Applied Sport Psychology, 8, 1-8.

Tenenbaum, G. (2001). A social-cognitive perspective of perceived exertion and exertion tolerance. In R.N. Singer, H.A. Hausenblas, \& C. Janelle (Eds.), Handbook of sport psychology (pp. 810-820). New York: Wiley \& Sons.

Ulmer, H.V. (1996). Concept of an extracellular regulation of muscular metabolic rate during heavy exercise in humans by psychophysiological feedback. Experientia, 52, 416-420. 
Walster, B., \& Aronson, E. (1967). Effect of expectancy of task duration on the experience of fatigue. Journal of Experimental Social Psychology, 3, 41-46.

Weinberg, R.S., Smith, J., Jackson, A., \& Gould, D. (1984). Effect of association, dissociation, and positive self-talk strategies on endurance performance. Canadian Journal of Applied Sport Science, 9, 25-32.

\section{Appendix}

\section{Associative and Dissociative Thoughts}

This experiment will monitor the types of thoughts you have while running. I will not want to know details of what you are thinking, only whether your thoughts are broadly associative or dissociative.

Associative Thoughts: Thoughts about your body and how it is reacting to the exercise, for example where your attention is focused on the body and your thoughts are about physical sensations such as heart pounding, sweatiness, heavy breathing, sore muscles, pain, etc.

Dissociative Thoughts: These are all thoughts that are not about physical sensations or the exercise. External thoughts are like daydreams and may distract you from the bodily signals arising from exercise.

Classify the following thoughts as associative or dissociative and then check at the bottom of the page to see if you got them correct.

1. "I'm feeling tired"

2. "I wonder how my friend is getting on in her new home."

3. "Breathe deeply, relax shoulders..."

4. "Just one more stretch to go and I can rest."

5. "Oh dear, I forgot to get some bread."

6. "Keep going, you can do it."

7. "That new sitcom is very funny."

8. "My feet are beginning to hurt."

Answers: Associative thoughts = 1, 3, 4, 6, 8; Dissociative thoughts = 2, 5, 7 .

\section{Acknowledgment}

We thank the ESRC for funding this research. Thanks also to Renee Pilbeam and the members of Totton Running Club for their help and participation, to Active Options Coordinator Paul Hedges, to Dave Cooper who helped with data collection, and to all who took part in Study 2. 